\title{
Location Privacy Online: China, The NETHERLANDS AND SOUTH KOREA
}

\author{
Peter Broeder ${ }^{1}$ and Yujin Lee ${ }^{2}$ \\ ${ }^{1}$ Communication and Information Sciences, Tilburg University, The Netherlands \\ ${ }^{2}$ Research Instruments, Falmouth, United Kingdom
}

\begin{abstract}
The aim of the study is to explore cross-cultural differences in users' location privacy behaviour on LBSNs (location-based social networks) in China, the Netherlands and Korea. The study suggests evidence that Chinese, Dutch and Korean users exhibit different location privacy concerns, attitudes to social influence, perceived privacy control and willingness to share location-related information on LBSNs. The results show that in general, the more concerned users are about location privacy, the less they are willing to share and it also suggests that location privacy concern and social influence affect each other. Furthermore, the more control people perceive they have over their privacy, the more they are willing to share location information. A negative relationship between willingness to share location information and users' actual sharing of location information was seen. In short, it is concluded that the relation between cultural values and location privacy behaviours only have a partial connection.
\end{abstract}

\section{KEYWORDS}

Privacy online, individualism, social influence, Asia, Europe

\section{INTRODUCTION}

Privacy is a basic and fundamental right and it is undeniable that privacy is deeply permeated throughout our everyday life. Privacy comprises many aspects so it is hard to describe with one unified definition, but generally it is defined as the claim of individuals, groups, or institutions to determine for themselves when, how, and to what extent information about them is communicated to others [1], or the capacity to control the information about oneself that is available to others [2].

As online social networks become more prevalent, the importance of information privacy increases. Social networks have given people the ability to form strong social ties by allowing them to connect with each other, to share personal information and, latterly, to share location, activities that all seem to raise privacy concerns. As the range of possible communications and interactions with others through social network channels has increased dramatically, privacy concerns have been mounting; our desire to take advantage of new interactive technologies has made us more willing to give up some of our privacy by sharing a large amount of personal information online.

Among the information we share in social networks, this study focuses on location information. A Location-based Service (LBS) depends on and is enhanced by positional information of mobile devices [3]. It provides users with opportunities to share geo-tagging content and real-time user location information. Little work has been done to explore the correlations between cultures and privacy concerns of users on Location-based Social Networks (LBSNs). Therefore, this research intends to examine how people from different cultures behave with respect to location privacy

DOI : $10.5121 /$ ijsptm.2016.5401 
concerns when using LBSNs. Accordingly, the study explores how Chinese, Dutch and Korean people use LBSNs and their location privacy concerns. Specifically, to what extent people reveal their location information, share with whom and how their concepts or awareness on location privacy differ.

\section{Privacy Online}

\subsection{LOCATION-BASED SOCIAL NETWORKS (LBSNS)}

LBSNs are mobile applications that allow people to build social networks and share their location with their friends or families. A number of major social network platforms have already launched its own featured location-based service. Facebook (Places, check-in etc.), Twitter (locating tweets), Foursquare (location check-in) and WeChat (People Nearby, Drift Bottle etc.) are those providing specific location-based functions. The number of LBSN providers has explosively increased in the past few years. There were more than 100 LBSN applications in 2010 [4]. The great popularity of LBS, however, carries the danger of user location privacy breaches due to location information disclosure. Location privacy is defined as the ability to prevent other parties from learning one's current or past location [5]. The privacy issue in location-based service can be seen as a core concept since it is a channel to broadcast users' real-time location. LBS users are concerned about loss of privacy and are worried about data or personal information collection on LBS [6].

Concerns over location privacy represent people's attitudes towards sharing location information. According to [7], over time, Internet users have become less concerned about information privacy. It is possible to assume that nowadays Internet users have knowledge to control privacy settings, thus, it leads to less concern about information privacy. Their research found that $71 \%$ of social networking users aged 18-29 in America have changed the privacy settings on their profiles to limit what they share with others online. Surprisingly, the most visible and engaged Internet users are most active in limiting the information connected to their names online. Therefore, it is presumable that people who are more likely to be concerned about privacy will share less location information on LBSN.

Hypothesis 1. People with more concerns about location privacy will be less willing to share location.

Social influence is defined as the degree to which individuals perceive that people who are important to them think they should use the service [8]. It is an equivalent term to subjective norm [9]. In a study conducted in an organizational environment [10] subjective norm is one of the factors that determine one's privacy intention. In addition, [11] also found that subjective norm is one of the factors which influence people's intentions to protect personal information privacy. More precisely, [12] showed that user's attitude toward social influence positively affects the use of WeChat. Hence, a hypothesis is composed:

Hypothesis 2. Social influence will have an impact on location based privacy concerns and willingness to share location.

People's confidence in their ability to perform certain behaviour can influence people's behavioural intention [9]. A study by [13] concluded that perceived control over personal information is the core factor that influence user information privacy. Following this perspective, it is presumable that users who think they have knowledge of managing and controlling privacy settings in an application or a service and may regard that they have high perceived privacy 
control, would result in increasing intentions to share personal information [14]. Therefore, a hypothesis is built:

Hypothesis 3. People with more perceived privacy control will be more willing to share location. Finally, this study proposes that willingness (intention) to share location based information positively leads to actual sharing of location based information.

Hypothesis 4. An increase in willingness to share location information will result in actual sharing of location.

\subsection{Cultural Differences}

Individualism/collectivism is one of the most used dimensions when it comes to understanding the way culture relates to social psychological phenomena. Individualism is described as a culture that reflects "I" autonomy, individual level of achievement or decisions are encouraged, and individuals have a relatively weak relationship bond. On the other hand, individuals in collectivist cultures reflect upon a "we" consciousness, prefer to behave as members of groups rather than individuals, [15] and [16]. Although the individualism/collectivism dimension has faced several criticisms (e.g., excessively polarization of cultural differences, assuming national uniformity, overlooking the possibility of individual differences [17]) it is still widely accepted and applied in cross-cultural studies [18].

Previous researches have shown fairly mixed results about the influence of individualism on privacy concerns or behaviours. [19] found a positive relationship between them while many other studies [20], [21] and [22], found it the opposite. Although many cross-cultural studies have generally found that cultures with high individualism are less concerned about information privacy, this study chooses the findings of [19] and posits that people in highly individualistic societies will be more likely to be concerned about information privacy, thus they will share location information on LBSN less than those in collectivistic cultures. This is based on the assumption that individuals in highly individualistic cultures value the right to a private life while people with lower scores on individualism are more likely to accept intrusion into their private life.

An important factor in deciding whether or not to share location or other information is with whom to share, [23] and [24]. In collectivistic, people in high power distance societies will be likely to share location information with classified groups. Power distance hereby means the extent to which the less powerful members of a society accept and expect unequally distributed power. China and Korea rank high in power distance whereas the Netherlands ranks low. Considering the highly hierarchical nature of high power distance cultures, it should be considered that people will exhibit different preferences on group orientation when sharing location information. In collectivistic cultures the difference in behaviours toward in-group and out-group members is very large, whereas in individualistic cultures it is greatly attenuated [17]. In this study, out-group particularly refers to the people who are in the range of a hierarchy structure (e.g., superiors, seniors) while in-group refers to family and friends in general. In addition, given the tendency that in high power distance societies, employees are not greatly encouraged to present their own opinions or ideas and generally do not have unconstrained conversations with superiors or seniors, it can be assumed that people in high power distance societies, mainly due to a hierarchical nature in organizations, seldom share private information with out-group members. 
Hypothesis 5. People from individualistic, low power distance cultures are more privacy concerned and less willing to share location compared to people in collectivistic high power distance cultures.

Most commonly, cross-cultural studies conceptualize that Western cultures tend to be individualistic with low power distance, while collectivism with high power distance is the basis of Asian cultures, [25] and [26]. However, a single 'Western' culture can comprise different levels of individualism/collectivism. [27] found that Americans showed lower scores on collectivism than Europeans. Also in Asian cultures there are different levels of individualism/collectivism characteristics among Asian countries. Specifically, Northeast Asian countries (China, Japan and Korea) exhibit low scores of individualism and higher scores on collectivism but their characteristics within individualism and collectivism are different while they share common cultural bonds, i.e., Confucian values. [28] also concluded that there is a significant difference of self-construal perspective among China, Japan and Korea and the result explained that Northeast Asian countries cannot be seen as a single oriental collectivism culture. This study hypothesizes that the Chinese culture is much more individualistic than the Korea culture based on the findings of [26], [29] and [30]. Therefore, it anticipates that Chinese users will be more reluctant to share location information.

Hypothesis 6. Chinese people are more privacy concerned and less willing to share location compared to Korean people.

\section{METHOD}

As a whole, the study aims at answering the following major research question: Do cultural differences play a role in users' location privacy behaviours and their privacy concerns on Location-Based Social Networks (LBSNs).

\subsection{SAMPLE}

In spring 2014, a total of 237 respondents completed an online survey: 128 females and 109 males, Mean age was 24.3 yrs (age range 18-35 yrs.). There were 52 Chinese, 108 Dutch, and 57 Korean respondents, mostly living in the pertinent countries that is in China, the Netherlands, and South Korea.

\subsection{QUESTIONNAIRE}

The questionnaire consisted of 23 statements (see Appendix).

- The subscale for location privacy concern (LPC) is defined as the ability to prevent other parties from learning one's current or past location [5] consists of five items. [14] and [31] are used as references.

- Perceived privacy control (PPC) is described as people's perception of the ease or difficulty of controlling or modifying privacy setting online [9]. The four items of this subscale were also built upon [14].

- Social influence (SI) is described as the degree to which individuals perceive that people who are important to them think they should use the system [12]. Based on [32] five items were developed.

- For in-group orientation (IG) a subscale consisting of six item was self-developed since 
no studies have yet been performed. In-group is often defined as "family and friends" [17]. In the context of this study, in-group orientation can be interpreted as a tendency of dividing groups (in-group which refers to friends and family and out-group which particularly refers to those who are in a range of the hierarchical structure such as superiors or seniors) and exhibiting different levels of preference to the groups. Six items were self-developed.

- Willingness to share (WS) is equivalent to intention [9]. Intentions are assumed to capture the motivational factors that influence behaviour; they are indications of how hard people are willing to try, of how much effort they are planning to exert, in order to perform the behaviour. [8] provided the idea for the two items of this scale.

- For the scale actual sharing of location information (AS) the ten items were based on the observation within Facebook and WeChat of what kind of location information users can share.

For the first four subscales the respondents were asked to rate the statements on a 5-point scale ranging from " $1=$ completely disagree" to " $5=$ completely agree". For the subscales Willingness to Share and Actual Sharing on Facebook/WeChat the respondents were asked to indicate yes or no.

\section{RESULTS}

\subsection{Internal Consistency OF The Subscales}

For each subscale the internal consistency was checked with Cronbach's $\alpha$. For Location privacy concern (LPC) $\alpha=.89$, for perceived privacy control (PPC) $\alpha=.82$, for willingness to share (WTS) $\alpha=.94$, for actual sharing (AS) on Facebook (FB) $\alpha=.70$, and for actual sharing (AS) on WeChat (WC) $\alpha=.85$. All were acceptable by Cronbach's $\alpha$ with higher than .70 . For the subscale social influence (SI) Cronbach's $\alpha$ was acceptable with $\alpha=.73$ (after elimination of item 5). The internal consistency of the subscale In-group orientation (IGO) was poor, with Cronbach's $\alpha=.53$ (after elimination of item four).

\subsection{LOCATION BASED PRIVACY INFORMation}

A considerable amount of location-related information is reported to be shared on Facebook and WeChat. Table 1 displays the actual percentage of shared location-related information.

Table 1. Shared Personal Profile Information on Facebook/Wechat.

\begin{tabular}{|l|l|r|r|}
\hline & Personal profile information & $\begin{array}{r}\text { Facebook } \\
(\mathrm{n}=159)\end{array}$ & $\begin{array}{r}\text { WeChat } \\
(\mathrm{n}=43)\end{array}$ \\
\hline \multirow{2}{*}{$\begin{array}{l}\text { Profile } \\
\text { information }\end{array}$} & Place of birth & $119(75 \%)$ & $19(44 \%)$ \\
\cline { 2 - 4 } & Place of residence & $135(85 \%)$ & $33(77 \%)$ \\
\cline { 2 - 4 } & Country of residence & $142(89 \%)$ & $35(81 \%)$ \\
\cline { 2 - 4 } & Home address & $7(4 \%)$ & $5(12 \%)$ \\
\hline \multirow{3}{*}{$\begin{array}{l}\text { Shared } \\
\text { information }\end{array}$} & Landmarks or well-known places & $82(52 \%)$ & $24(56 \%)$ \\
\cline { 2 - 4 } & Restaurants & $69(43 \%)$ & $23(53 \%)$ \\
\cline { 2 - 4 } & Vacation or holiday destinations & $93(58 \%)$ & $24(56 \%)$ \\
\cline { 2 - 4 } & Other locations where I would like to check-in & $54(34 \%)$ & $13(30 \%)$ \\
\hline
\end{tabular}

Places of birth, residence and home address can be displayed on a personal profile while the rest of the items can be seen by 'sharing'. One thing which should be noted is that only a small 
number of users shared their home address information (7/159 Facebook users and 5/43 WeChat users). Considering users tend to share a lot of other information other than home address, it can be interpreted that home address can be regarded as one of the most private among the range of location-related information, hence, it is possible to interpret that people are actually concerned strongly about their location privacy. A great number of users shared place of birth, residence and country of residence. It seems that because the information does not show details of the exact location or place but just a country of birth, a city and country of residence in general, users do not mind sharing the information very much. Table 2 shows the results of the correlation analysis for the factors of users' privacy behaviour.

Table 2. Correlation Analysis on the Factors of Users' Privacy Behaviour.

\begin{tabular}{|l|ll|ll|lll|}
\hline & LPC & PPC & SI & IGO & WTS & AS FB & AS WC \\
\hline Location privacy concem(LPC) & 1 & .028 & $.467^{* *}$ & $.529 * *$ & $-.242^{* *}$ & .066 & .231 \\
Perceived privacy control (PPC) & & 1 & .003 & .080 & $.337^{* *}$ & $-.212^{* *}$ & $.344^{*}$ \\
\hline Social Influence(SI) & & & 1 & $.371^{* *}$ & $-.228^{* *}$ & $.246^{* *}$ & .200 \\
In-group orientation(IGO) & & & & 1 & -.021 & .044 & -.080 \\
\hline Willingness to share(WTS) & & & & & 1 & $-.401^{* *}$ & $-.369^{*}$ \\
Actual sharing Facebook (AS FB) & & & & & & 1 & $.657^{* *}$ \\
Actual sharing WeChat (AS WC) & & & & & & & 1 \\
\hline
\end{tabular}

$* \mathrm{p}<.05, * * \mathrm{p}<.01$

Hypothesis 1 predicted that people who are more concerned about location privacy will be less willing to share location information. A negative relationship was found between location privacy concern and willingness to share $(\mathrm{r}=-.242$, Bca 95\% CI $[-.405,-.068], \mathrm{p}<.005)$.

This study also assumed the impact of social influence (Hypothesis 2). As can be interpreted in Table 2. social influence and willingness to share location information have negative significant correlations $(\mathrm{r}=-.228$, Bca 95\% CI [-.364, -.085], $\mathrm{p}<.005)$. From this result, it is predictable that people who are more sensitive about their friends or families' opinions are less willing to share location information. Similarly, the correlation analysis shared that location privacy concern is positively linked to social influence $(r=.467$, Bca 95\% CI $[.338, .570], \mathrm{p}<.001)$.

The more perceived privacy control people have, the more they are willing to share location information (Hypothesis 3). This was indeed confirmed by the observed positive relationship between perceived privacy control and willingness to share location information $(r=.337$, Bca $95 \%$ CI $[.174, .480], \mathrm{p}<.001)$. It was also assumed that escalation of intention would result in an increase of actual behaviour (Hypothesis 4). In the current study, respondents' willingness to share location information and actual sharing on Facebook had a significant correlation, $r=-.401$, Bca 95\% CI [-.524, -.275], $\mathrm{p}<.001$, however, it is negatively correlated, hence, more willingness actually resulted in less sharing of location-related information. The case of WeChat shows a similar result as Facebook ( $r=-.369$, Bca 95\% CI [-.633, -.063], $\mathrm{p}<.05)$.

\subsection{Privacy Preferences Of Chinese, Dutch And Koreans}

We theorized that relationships exist between cultural preferences and location privacy behaviour. Hypotheses 5 and 6 inferred that Chinese, Dutch and Koreans will exhibit different location privacy concerns, perceived privacy control and attitudes toward social influence. Table 3 and Figure 1 show the three groups' scores for each factor. 
Table 3. Privacy Behaviour Per Cultural Group: Mean Of A 5-Point Scale (Minimum=1, Maximum=5) and Standard Deviation (SD).

\begin{tabular}{|l|rc|rc|cc|}
\hline & \multicolumn{2}{|c|}{$\begin{array}{c}\text { Chinese } \\
\text { (n=45) }\end{array}$} & \multicolumn{2}{c|}{$\begin{array}{c}\text { Korean } \\
(\mathrm{n}=43)\end{array}$} & \multicolumn{2}{c|}{$\begin{array}{c}\text { Dutch } \\
(\mathrm{n}=91)\end{array}$} \\
\hline Location privacy concem(LPC) & 3.90 & $(.80)$ & 3.85 & $(.87)$ & 3.18 & $(.88)$ \\
Perceivedprivacy control (PPC) & 3.60 & $(.72)$ & 3.41 & $(.93)$ & 3.52 & $(.78)$ \\
\hline Social Influence(SI) & 3.44 & $(.97)$ & 3.57 & $(1.06)$ & 3.33 & $(1.04)$ \\
In-group orientation(IGO) & 3.19 & $(.57)$ & 3.28 & $(.66)$ & 3.01 & $(.48)$ \\
\hline Willingness to share(WTS) & 3.35 & $(1.17)$ & 2.97 & $(.92)$ & 3.34 & $(.97)$ \\
\hline Actual sharing Facebook(AS FB) & 1.45 & $(.21)$ & 1.45 & $(.24)$ & 1.50 & $(.21)$ \\
Actual sharing WeChat (AS WC) & 1.50 & $(.30)$ & 1.65 & $(.26)$ & - & $(-)$ \\
\hline
\end{tabular}

A one way between subjects ANOVA was conducted to compare the level of location privacy concerns, perceived privacy control and attitudes toward social influence. For location based privacy concern, significant differences were shown for the three conditions $[F(2,170)=14.062$, $\mathrm{p}<.001]$. Post hoc comparisons using the Tukey HSD test indicated that the mean scores for the Chinese group $(\mathrm{M}=3.90, \mathrm{SD}=0.80)$ and Korean group $(\mathrm{M}=3.85, \mathrm{SD}=0.87)$ were significantly higher than those for the Dutch group $(\mathrm{M}=3.18, \mathrm{SD}=0.88)$. However, the Chinese and Korean groups did not significantly differ. Remarkably, both perceived privacy control and influence did not display significant differences between the three cultural groups.

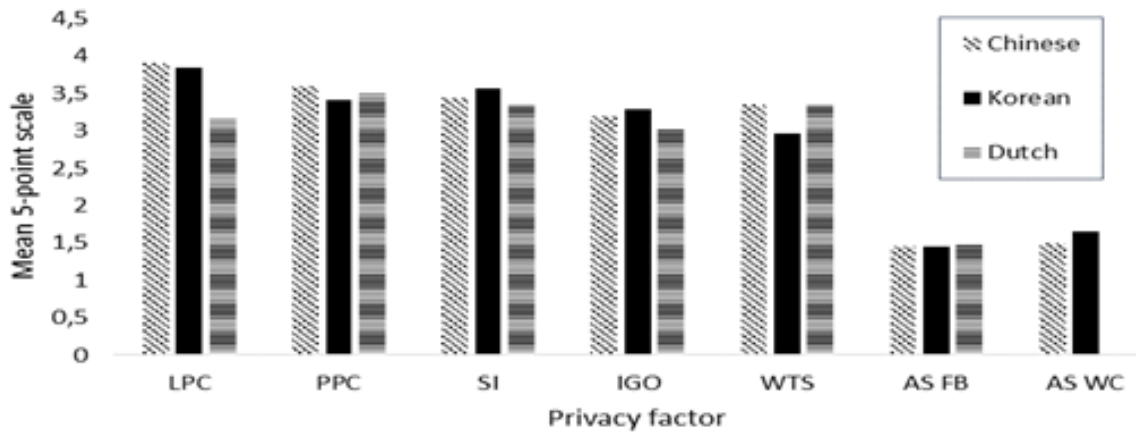

Figure 1. Privacy Behaviour Per Cultural Group.

Hypothesis 6 implied that people from collectivistic, high power distance societies are more likely to share location information. As Table 3 and Figure 1 shows, the highest score on power distance (SI) was seen within the Korean group. However their willingness to share location information (WTS) is not as high as the Chinese and Dutch, rather it shows the lowest score.

Power distance can also be linked to in-group orientation (IGO). People in countries with higher scores of power distance will exhibit clear in-group orientation compared to people in low power distance societies when sharing location information. Koreans who had the highest scores in power distance also showed the highest in-group orientation tendency. Finally, the study hypothesized that people in countries with higher scores on individualism are less likely to share location information. However, since no significant differences were found on individualism, it is not measureable yet.

\section{CONCLUSIONS}

Online privacy is deeply entwined with our daily life. Among various areas of online privacy, location privacy can be regarded as one of the most 'private' areas. The general results suggest 
that LBSN users have great concern over their location privacy, and the more users are concerned the less willing they are to share location information. The findings in this study show that users' attitudes toward social influence have an impact on the willingness to share. People who are sensitive about their family or friends opinions, are less willing to share location information. Similarly, the more perceived privacy control users have, the more they are willing to share. In the meantime, location privacy concern and social influence affect each other.

Finding possible link between users' location privacy behaviour and cultural values was one of the purposes of the study. It was assumed that Chinese and Korean users would be more likely to exhibit collectivistic behaviour and high power distance cultures, and that Chinese users would exhibit higher collectivism than Korean. The key reference of the study [22] claimed that crosscultural dimensions are significant predictors of information privacy concerns and desire for online awareness in particular uncertainty avoidance, power distance and individualism dimensions. Unlike the study of [22], the present research did find only limited links between location privacy behaviour and cultural values. The result only found that people in higher scores of power distance societies exhibit clear in-group orientation compared to those in low power distance societies when sharing location. The relation between cultural values and location privacy behaviours require more comprehensive future research.

Overall, the study has brought empirical evidence on users' location privacy behaviour. It revealed that factors of location privacy concern, social influence and perceived privacy control are embedded in user's behaviour on LBSNs. In particular, the study found a significant relationship between users' location privacy concern and intention to share. What makes this study more valuable is a cross-cultural comparison. Considering no study has been done on the relationship between location privacy and cultural differences so far, the study could trigger more future research on this or similar topics.

\section{ACKNOWLEDGEMENTS}

We would like to thank colleagues of the research group "Online consumers" for all feedback and discussions, as well as the anonymous reviewers, for their helpful comments.

\section{REFERENCES}

[1] Westin, A. (1967). Privacy and Freedom. New York: Atheneum

[2] Stone, E. F., Gueutal, H., Gardner, D. \& McClure, S. (1983). A field experiment comparing information-privacy values, beliefs, and attitudes across several types of organizations. Journal of Applied Psychology 68, 459-468.

[3] Dhar, S., \& Vahrsney, U. (2011). Challenges and business models for mobile location-based services and advertising. Communications of the ACM 54(5), 121-129

[4] Fusco, S. J., Michael, K., \& Michael, M. (2010). Using a social informatics framework to study the effects of location-based social networking on relationships between people: A review of literature. IEEE International Symposium on Technology and Society (ISTAS), 157-171.

[5] Beresford, A. R., \& Stajano, F. (2003). Location privacy in pervasive computing. IEEE Pervasive Computing 2(1), 46-55.

[6] Webroot (2010), Survey Finds Geolocation Apps Prevalent Amongst Mobile Device Users, But 55\% Concerned About Loss of Privacy (www.webroot.com).

[7] Madden, M., \& Smith, A. (2010). Reputation management and social media. Pew Internet \& American Life Project. Pew Research Center (www.pewinternet.org).

[8] Venkatesh, V., Morris, M., Davis, G., \& Davis, F. (2003). User acceptance of information technology: Toward a unified view. MIS Quarterly 27, 425-478.

[9] Ajzen, I. (1991). The Theory of Planned Behavior. Organizational Behavior and Human Decision Processes 50, 179-211. 
[10] Loch, K. \& Conger, S. (1996). Evaluating ethical decision making and computer use. Communications of the ACM 39 (7), 74-83.

[11] Hsu, M. \& Kuo, F. (2003). The effect of organization-based self-esteem and deindividuation in protecting personal information privacy. Journal of Business Ethics 42, 305-320.

[12] Zeng, Z., Hu, X. \& Mei, S. (2013). Factors affecting a mobile application's acceptance: An empirical study of user acceptance of WeChat in China. Jonkoping International Business School, Jonkoping University.

[13] Xu, H., Teo, H., Tan, B. \& Ritu A. (2012). Effects of individual self-protection, industry selfregulation, and government regulation on privacy concerns: A study of location-based services. Information Systems Research 23, 1342-1363.

[14] Notermans, R. (2013). To share or not to share? The personal aspects of online privacy behavior. Tilburg University.

[15] Triandis, H., Bontempo, R., Villareal, M., Asai, M. \& Lucca, N. (1988). Individualism and collectivism: Cross-cultural perspectives on self-ingroup relationships. Journal of Personality and Social Psychology 54, 323-338.

[16] Hofstede, G. (2001). Culture's consequences: Comparing values, behaviors, institutions, and organizations across nations. Thousand Oaks, CA: Sage.

[17] McSweeney, B. (2002). Hofstede's model of national cultural differences and their consequences: A triumph of faith - a failure of analysis, Human Relations 55, 89-118.

[18] Hofstede, G. (2002). Dimensions do not exist: A reply to Brendan McSweeney. Human Relations 55, 1355-1361.

[19] Milberg, S., Smith, J. \& Burke, S., (2000). Information privacy: Corporate management and national regulation. Organization Science 11, 35-57.

[20] Ting-Toomey, S. (1991). Intimacy expressions in three cultures: France, Japan, and the United States. International Journal of Intercultural Relations 15, 29-46.

[21] Bellman, S., Johnson, E., Kobrin, S., \& Lohse, G. (2004). International differences in information privacy concerns: A global survey of consumers. The Information Society 20(5), 313-324.

[22] Lowry, P., Cao, J., \& Everard, A. (2011). Privacy concerns versus desire for interpersonal awareness in driving the use of self-disclosure technologies: The case of instant messaging in two cultures. Journal of Management Information Systems 27(4), 163-200.

[23] Consolvo, S., et al. (2005). Location disclosure to social relations: why, when, \& what people want to share. Proceedings of the SIGCHI conference on human factors in computing systems, 81-90.

[24] Wiese, J., et al. (2011). Are you close with me? Are you nearby? Investigating social groups, closeness, and willingness to share. Proceedings of the 13th international conference on ubiquitous computing 197-206.

[25] Kitayama, S., Markus, H., Matsumoto, H. \& Norasakkunkit, V. (1997). Individual and collective process in the construction of the self: Self-enhancement in the United States and self-criticism in Japan. Journal of Personality and Social Psychology 72, 1245-1267.

[26] Herrmann-Pillath, C. (2010). Social capital, Chinese style: individualism, relational collectivism and the cultural embeddedness of the institutions-performance link. China Economic Journal 2, 325-350.

[27] Oyserman, D., Coon, H. \& Kemmelmeier, M. (2002). Rethinking individualism and collectivism: Evaluation of theoretical assumptions and meta-analyses. Psychological Bulletin 128, 3-72.

[28] Han, M., Yoshiyuki I., Kim, S. \& Zhang, W. (2009). The application of culture bounded selfconstrual model: A comparative study between three countries in Northeast Asia. Journal of Korean Psychology Association 28(1), 49-66.

[29] Kwon, J. \& Oh, D. (2010). An examination of thirty-year cross-cultural research in Korea: the case of Hofstede's cultural values. International Business Management Review 14 (4), 1-32.

[30] Li, H., Zhang, Z., Bhatt, G., \& Yum, Y. (2006). Rethinking culture and self-construal: China as a middle land. The Journal of Social Psychology 146, 591-610.

[31] Buchanan, T., Paine, C., Joinson, A. \& Reips, U. (2007). Development of measures of online privacy concern and protection for use on the internet. Journal of the American Society for Information and Science and Technology 58, 157-165.

[32] Chen, R. Wang, J., Heratch, T. \& Rao, H. (2011). An investigation of email processing from a risky decision making perspective. Decision Support Systems 52, 73-81. 


\section{AUTHORS}

Peter Broeder investigates intercultural aspects of online marketing communications and consumer behaviour. These issues are addressed in Europe and Asia (www.broeder.com).

Yujin Lee obtained her master's degree from Tilburg University (the Netherlands) in 2014. Currently she is a Business Development Manager at Research Instruments, Falmouth (United Kingdom).
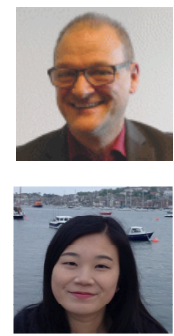

\section{APPENDIX}

Location Privacy Concern (LPC)

I am concerned about my privacy in general while I am using the Internet.

I am concerned about my privacy in general while I am posting something on social media.

I am concerned about people I don't know obtaining my personal information from my online activities.

I am concerned about sharing location on social media.

I am concerned about people who I don't know accessing my location information from social media.

Perceived Privacy Control (PPC)

In general, I know HOW to modify my privacy setting on Facebook/WeChat.

It is easy to modify WHAT kind of location information is being presented on Facebook/WeChat via privacy setting.

I am confident that I can control WHOM I share location on Facebook/WeChat with.

I am confident that I can control WHEN to share location on Facebook/WeChat.

Social Influence (SI)

People who are important to me think that sharing location on Facebook/WeChat is very risky.

People who influence my behaviour think using location-based social media is very risky.

I really don't mind sharing location information with everybody.

People whose opinions I value think sharing location on Facebook/WeChat is highly risky.

(I use Facebook/WeChat because many of my friends and colleagues use it)*.

In-group Orientation (IG)

When I post something on Facebook/WeChat, I usually select groups whom I want to share with.

When I share my location on Facebook/WeChat, I would not be worried if anyone can access my information.

I want to share my location information only with friends.

(The reason why I check-in on Facebook/WeChat is because I want to share the location with my friends)*. I don't want my superiors to see all my location information on Facebook/WeChat.

If my superiors see those locations that I share on Facebook/WeChat, I would worry that it might have negative effects on me.

Willingness to Share (WTS)

I am willing to keep using Facebook/WeChat when sharing location for the next 12 months.

I plan to keep using Facebook/WeChat when sharing location information for the next 12 months.

Actual Sharing on Facebook/WeChat (AS)

Place of birth. Place of residence. Country of residence. Home address. Landmarks or well-known places. Restaurants. Vacation or holiday destinations (airport, accommodation, etc.). Random locations where I would like to check-in. Places where I haven't been. Tagging friends who are with me on the shared location or check-in.

*Items deleted after reliability analyses (Cronbach's $\alpha$ ) 